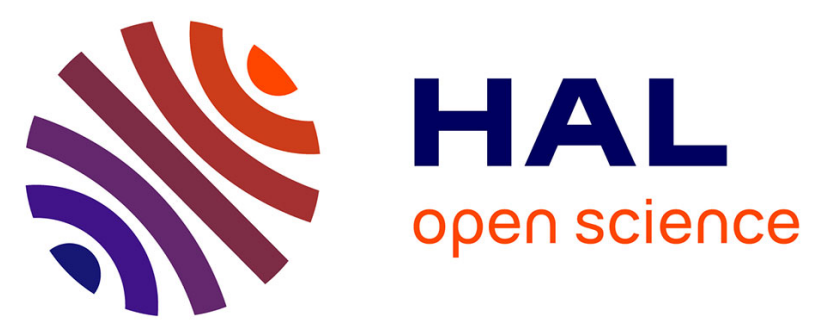

\title{
Characterizing the peritumoral brain zone in glioblastoma: a multidisciplinary analysis.
}

Jean-Michel Lemée, Anne Clavreul, Marc Aubry, Emmanuelle Com, Marie de Tayrac, Pierre-Antoine Eliat, Cécile Henry, Audrey Rousseau, Jean Mosser, Philippe Menei

\section{To cite this version:}

Jean-Michel Lemée, Anne Clavreul, Marc Aubry, Emmanuelle Com, Marie de Tayrac, et al.. Characterizing the peritumoral brain zone in glioblastoma: a multidisciplinary analysis.. Journal of NeuroOncology, 2015, 122 (1), pp.53-61. 10.1007/s11060-014-1695-8 . hal-01117083

\section{HAL Id: hal-01117083 \\ https://hal-univ-rennes1.archives-ouvertes.fr/hal-01117083}

Submitted on 16 Jun 2015

HAL is a multi-disciplinary open access archive for the deposit and dissemination of scientific research documents, whether they are published or not. The documents may come from teaching and research institutions in France or abroad, or from public or private research centers.
L'archive ouverte pluridisciplinaire HAL, est destinée au dépôt et à la diffusion de documents scientifiques de niveau recherche, publiés ou non, émanant des établissements d'enseignement et de recherche français ou étrangers, des laboratoires publics ou privés. 


\title{
Characterizing the Peritumoral Brain Zone in
}

\section{Glioblastoma: a Multidisciplinary Analysis}

\author{
Jean-Michel LEMÉE ${ }^{1,2}$, M.D. \\ Anne CLAVREUL ${ }^{1,2}$, Ph.D. \\ Marc AUBRY ${ }^{3}$, Ph.D. \\ Emmanuelle COM ${ }^{4}$, Ph.D. \\ Marie DE TAYRAC ${ }^{3}$, M.D., Ph.D. \\ Pierre-Antoine ELIAT ${ }^{5}$, Ph.D. \\ Cécile HENRY ${ }^{6}$, M.Sc. \\ Audrey ROUSSEAU ${ }^{2,7}$, M.D., Ph.D. \\ Jean MOSSER ${ }^{3}$, M.D., Ph.D. \\ Philippe MENEI ${ }^{1,2}$, M.D., Ph.D.
}

${ }^{1}$ Département de Neurochirurgie, Centre Hospitalier Universitaire d'Angers, 4 rue Larrey, 49933 Angers Cedex 09, France

2 INSERM UMR-1066, Micro- et Nanomédecine Biomimétiques (MINT), 4 rue Larrey, 49933 Angers Cedex 09, France

${ }^{3}$ Plate-forme Génomique Santé Biosit, Université Rennes1, 35043 Rennes, France

${ }^{4}$ Proteomic Core Facility BiogenOuest, IRSET, Inserm U1085, Campus de Beaulieu, 35042 Rennes, France

${ }^{5}$ PRISM, SFR Biosit, Université de Rennes 1, CNRS UMS 3480 - INSERM US 018, 35043 Rennes, France

${ }^{6}$ Centre Régional de Lutte contre le Cancer Paul Papin, 2 rue Moll, 49000 Angers, France

${ }^{7}$ Département de Pathologie Cellulaire et Tissulaire, Centre Hospitalo-universitaire d'Angers, 4 rue Larrey, 49933 Angers Cedex 09, France

Running Title: Analysis of glioblastoma peritumoral brain zone

Corresponding author:

Pr Philippe Menei, M.D., Ph.D.

Department of Neurosurgery

4, rue Larrey 49933 Angers Cedex 09

Tél: $0033(0) 241353977$

Fax: 0033 (0)2 41354508

Mail: phmenei@ chu-angers.fr

Keywords: Glioblastoma, peritumoral brain zone, genomics, transcriptomics, proteomics, histopathology

Running head: Glioblastoma peritumoral brain zone: a multimodal analysis

Financial disclosure:

This work was supported by the Cancéropôle Grand Ouest and the Institut National du Cancer (INCa).

The first author of the study (J.-M.L.) received grants from the Société Française de Neuro-Chirurgie (SFNC) and from the Institut National de la Santé et de la Recherche Médicale (INSERM).

This work will be presented as a poster at the Congress of the French speaking association of Neuro-Oncologist (ANOCEF), May 17 $7^{\text {th }}$ 2014, in Lausanne, Swiss and as an oral communication in the Congress of the French society of Neurosurgery, May $23^{\text {rd }}$, in Clermont-Ferrand, France 


\section{Abstract}

Glioblastoma (GB) is the most frequent and aggressive type of primary brain tumor. Recurrences are mostly located at the margin of the resection cavity in the peritumoral brain zone (PBZ). Although it is widely believed that infiltrative tumor cells in this zone are responsible for GB recurrence, few studies have examined this zone.

In this study, we analyzed PBZ left after surgery with a variety of techniques including radiology, histopathology, flow cytometry, genomic, transcriptomic, proteomic, and primary cell cultures. The resulting PBZ profiles were compared with those of the GB tumor zone and normal brain samples to identify characteristics specific to the PBZ. We found that tumor cell infiltration detected by standard histological analysis was present in almost one third of PBZ taken from an area that was considered normal both on standard MRI and by the neurosurgeon under an operating microscope. The panel of techniques used in this study show that the PBZ, similar to the tumor zone itself, is characterized by substantial inter-patient heterogeneity, which makes it difficult to identify representative markers. Nevertheless, we identified specific alterations in the PBZ such as the presence of selected tumor clones and stromal cells with tumorigenic and angiogenic properties.

The study of GB-PBZ is a growing field of interest and this region needs to be characterized further. This will facilitate the development of new, targeted therapies for patients with GB and the development of approaches to refine the per-operative evaluation of the PBZ to optimize the surgical resection of the tumor.

Keywords: Glioblastoma, peritumoral brain zone, genomics, transcriptomics, proteomics, histopathology 


\section{Introduction}

Glioblastoma $(\mathrm{GB})$ is the most frequent and aggressive type of primary tumor in the central nervous system (CNS)[1]. The standard treatment is surgical resection followed by fractionated radiotherapy, with concomitant and adjuvant chemotherapy with temozolomide[2]. Despite this treatment, the prognosis remains poor with a mean progression-free survival of seven months and an average overall survival of 15 months[1].

Gross total resection of GB, which is the first step of therapeutic management, has a major effect on overall survival, progression-free survival, and the quality of life of the patient $[3,4]$. It is only achieved in 35 to $50 \%$ of cases $[5,6]$ because the presence of functional areas makes it impossible to perform a resection with safe margins and no risk of neurological impairment [4]. Despite complete surgical resection, recurrence happens in almost all cases, mostly at the margin of the resection cavity in the peritumoral brain zone (PBZ) [7, 8]. It is widely believed that tumor cell infiltration into the PBZ may promote recurrence [9-11]. However, few cellular and molecular analyses have been performed in this area. Some studies have shown that residual, unresected tumor cells display alterations different from those of cells isolated from the corresponding tumor mass [12-14]. A better understanding of the characteristics of the PBZ and tumor cell infiltration in this region is critical to unravel the mechanisms underlying the recurrence of GB and to optimize the quality of surgical resection and the development of new therapies.

As part of the "Grand Ouest Glioma Project", funded by the French National Institute of Cancer, whose objective was to study tumor heterogeneity in GB [15-22], we performed a multidisciplinary analysis of the PBZ with a variety of techniques including radiology, histopathology, flow cytometry, omic analyses and primary cell cultures. The resulting PBZ profiles were compared with those of GB tumor zone (TZ) and normal brain samples to identify characteristics specific to the PBZ. 


\section{Material and methods}

\section{Patient recruitment and brain biopsies}

Thirty-nine patients with de novo GB were included in the "Grand Ouest Glioma Project". All patients gave informed consent prior to their enrolment. The study protocol was approved by the local ethics committee (CPP Ouest II, Angers, France).

Image-guided neuronavigation (Brainlab®, La Défense, France) was used during pre-surgical planning to define four regions of interest (ROI) in the tumor and its surroundings: the PBZ, the interface zone (IZ), the florid tumor zone (TZ), and the central necrotic zone (NZ) (Figure 1A). Biopsies were performed in the selected regions and were studied by various techniques: radiology, histopathology, flow cytometry, omic, and cell culture analyses and the results of these various methods were compared. All types of analysis could not be performed for each PBZ sample due to limited sample size. Among the 39 patients initially included, biopsies from 28 patients were analyzed by at least three techniques.

\section{Radiological analysis}

Standardized sequences were used for pre-surgical MRI and were carried out in the following order: T1-weighted axial and coronal acquisitions, diffusion, a quantitative dynamic contrast-enhanced (DCE) session after the injection of gadolinium at a concentration of $0.1 \mathrm{mmol} / \mathrm{kg}$, and finally T2 FLAIR, T2-weighted and 3D T1 acquisitions. For a detailed description of the calculation of DCE-MRI variables in all four zones (NZ, TZ, IZ and PBZ), see Supplementary Methods.

\section{Histolopathogical analysis}

For histopathological analysis, formalin-fixed paraffin-embedded sections of the biopsy specimens were stained with hematoxylin-phloxin-saffron (HPS). The diagnosis of GB was made according to the 2007 WHO classification of CNS tumors [23].

\section{Flow cytometry analysis}

DNA of cells of the PBZ and those of the TZ from 25 patients was stained by Vindeløv's protocol [24] and the samples were analyzed by flow cytometry (BD Biosciences, Le Pont de Claix, France). DNA index (DI) and the percentage of cell populations identified on the basis of different DNA contents were calculated with Modfit version 5.2 software (Verity Software House, Topsham, Maine) as previously described [15].

\section{Omic analyses}


PBZ and TZ samples were used for genomic $(n=10)$, transcriptomic $(n=9)$ and proteomic $(n=6)$ analyses. Brain samples from patients who underwent epilepsy surgery were also used as control brain samples. For a detailed description of omic analyses, see Supplementary Methods.

\section{B}

A

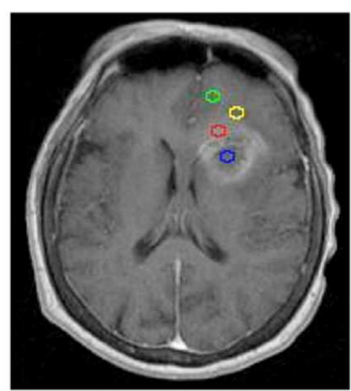

\begin{tabular}{|c|c|c|c|c|c|c|c|c|c|c|}
\hline PBZ & $\begin{array}{l}K^{\text {trans }} \\
\left(s^{-1}\right)\end{array}$ & $\begin{array}{l}V_{e} \\
(\%)\end{array}$ & $\begin{array}{l}\mathrm{s0} \\
\left(\mathrm{s}^{-2}\right)\end{array}$ & $\begin{array}{l}\mathbf{s 3 0} \\
\left(\mathrm{s}^{-2}\right)\end{array}$ & $\begin{array}{l}s 60 \\
\left(s^{-2}\right)\end{array}$ & $\begin{array}{l}s 360 \\
\left(s^{-2}\right)\end{array}$ & $\underset{\left(s^{-1}\right)}{\Delta R 1 \max }$ & $\begin{array}{l}\text { TTP } \\
\text { (s) }\end{array}$ & $\begin{array}{l}\text { AUC } \\
\text { (ua) }\end{array}$ & $\begin{array}{c}\mathrm{T} 10 \\
\text { (ms) }\end{array}$ \\
\hline GB-03 & 0.0305 & 0.026 & 0.0015 & 0.0008 & 0.0004 & 0 & 0.068 & 160 & 43 & 1120 \\
\hline GB-09 & 0.055 & 0.0362 & 0.0028 & 0.0012 & 0.0005 & 0 & 0.096 & 130 & 78 & 1204 \\
\hline GB-10 & 0.0174 & 0.0099 & 0.0009 & 0.0003 & 0.0001 & 0 & 0.027 & 116 & 22 & 1113 \\
\hline GB-11 & 0.006 & 0.0078 & 0.0003 & 0.0002 & 0.001 & 0 & 0.019 & 217 & 16 & 1289 \\
\hline GB-21 & 0.003 & 0.0208 & 0 & 0 & 0 & 0 & 0.008 & 835 & 4 & 1139 \\
\hline GB-22 & 0.0305 & 0.0009 & 0.0003 & 0.0001 & 0 & 0 & 0.007 & 57 & 1 & 970 \\
\hline GB-26 & 0.0183 & 0.0067 & 0.0008 & 0.0004 & 0.0001 & 0 & 0.026 & 74 & 14 & 1098 \\
\hline Mean PBZ & 0.022 & 0.013 & 0.0009 & 0.0004 & 0.0002 & 0 & 0.036 & 227 & 25.4 & 1133 \\
\hline Mean IZ & 0.113 & 0.097 & 0.0042 & 0.0022 & 0.0009 & 0 & 0.243 & 257 & 167.6 & 1151 \\
\hline Mean TZ & 0.299 & 0.271 & 0.0134 & 0.0068 & 0.0028 & 0.0001 & 0.676 & 365 & 484.4 & 1352 \\
\hline Mean NZ & 0.005 & 0.035 & 0.0002 & 0.0002 & 0.0001 & 0 & 0.059 & 817 & 50.7 & 1517 \\
\hline $\begin{array}{l}\text { Multivariate } \\
\text { Analysis }\end{array}$ & $<0.001$ & 0.001 & $<0.001$ & $<0.001$ & 0.003 & 0.167 & $<0.001$ & 0.006 & 0.001 & 0.061 \\
\hline $\begin{array}{c}\text { Univariate } \\
\text { Analysis } \\
\text { PBZ vs.TZ }\end{array}$ & 0.086 & $<0.001$ & 0.066 & 0.024 & 0.003 & 0.126 & 0.001 & 0.331 & 0.001 & 0.063 \\
\hline
\end{tabular}

\section{Fig. 1:}

A) Example of pre-surgical planning of biopsies on an axial contrast-enhanced T1 MRI brain slide. Blue area: necrotic zone (NZ), red area: florid tumor zone (TZ), yellow area: intermediate zone (IZ) and green area: peritumoral brain zone (PBZ)

B) Individual results for quantitative DCE MRI analysis of PBZ and comparison to the other sampling areas (IZ, $\mathrm{NZ}$ and $\mathrm{TZ})\left(\underline{\mathrm{K}^{\text {trans}}}\right.$ : permeability constant, $\underline{\mathrm{V}}_{\mathrm{e}}$ : fractional volume of extravascular extracellular space, $\underline{\mathrm{s} 0:}$ initial

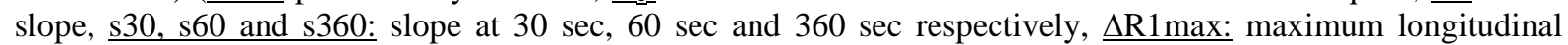
relaxation rate variation, TTP: time to peak, $\underline{\text { AUC: }}$ area under the curve, T1 $\underline{0}$ : longitudinal relaxation time before gadolinium injection).

\section{Primary cultures}

PBZ from 19 patients were used for cell cultures. Biopsy specimens were minced and mechanically dissociated in Dulbecco's modified Eagles' medium-high glucose (DMEM-HG) (Lonza, Verviers, Belgium). The resulting suspension was seeded in T80 flasks (Nunc, Dominique Dutscher, Brumath, France) containing DMEM-HG supplemented with 10\% human AB serum (EFS, Lyon, France) and 1\% antibiotics (Sigma-Aldrich, Saint Quentin Fallavier, France). Cells were grown at $37^{\circ} \mathrm{C}$ in a humidified incubator, under an atmosphere containing $5 \% \mathrm{CO}_{2}$, and the medium was changed twice weekly. Contrast-phase microscopy was used to examine cells at passage 1 or 2 . 


\section{Results}

\section{Radiological analysis}

We examined NZ, TZ, IZ and PBZ of seven patients with GB by DCE-MRI.

The comparison of the four zones in a multivariate analysis shows significant differences in all variables, except for $\mathrm{s} 360$ and $\mathrm{T} 1_{0}$ (Figure 1B). The PBZ presents lower values of $\mathrm{k}^{\text {trans }}$ and $\mathrm{v}_{\mathrm{e}}$ than the $\mathrm{TZ}$, indicating that the blood-brain barrier was preserved in the PBZ, in contrast to the TZ. Contrast enhancement was also lower in the PBZ than in the $\mathrm{TZ}$ and the AUC of contrast enhancement over time was significantly lower in the PBZ. We found that PBZ enhancement curves were consistent, except for patient GB-09. All constants were high for this patient, although they were not statistically different from those of other samples.

These results suggest that the DCE-MRI properties of the PBZ were similar to those of normal brain previously described by Tofts [25]. This was expected because PBZ was defined in our study as a radiologically normal peritumoral tissue, located at a distance from the brain/tumor interface, with the absence of gadolinium enhancement and a normal aspect on T1-weighted sequences.

\section{Histopathological analysis}

All PBZ samples were considered macroscopically similar to normal brain under surgical microscope magnification. Histological analysis showed no abnormalities for 20 of the 28 biopsies (71\%). We observed mild tumor cell infiltration in six patients $(21 \%)$ and marked infiltration in two patients (7\%) (Supplementary Table $1)$.

\section{Flow cytometry analysis}

Analysis of DNA index by flow cytometry is highly informative only if the tumor is aneuploid. We analyzed PBZ and TZ samples from 25 patients; eight (32\%) did not contain an aneuploid cell population, thus flow cytometry could not be applied to assess tumor cell infiltration in these PBZ. Among the 17 patients with an aneuploid cell population in their TZ, eight (47\%) displayed aneuploid cells in the PBZ and the percentage of aneuploid cells in the PBZ ranged from 3 to $44 \%$ (Table 1).

Interestingly, for a few samples (GB04, GB-09, GB-10 and GB-12), the PBZ contained only a few of the aneuploid cell populations identified in the TZ. This suggests that some tumor clones, but not all, migrate away from the tumor core.

Histopathology and flow cytometry gave similar results because all PBZ with a normal flow cytometry profile were considered free of tumor cell infiltration by histopathological analysis. However, flow cytometry 
was more a sensitive approach to detect tumor cell infiltration than histopathological analysis, because three PBZ with an aneuploid cell population were identified as histologically normal (GB-06, GB-09, and GB-12).

Table 1: DNA index of TZ and their corresponding PBZ. All TZ presented in this table contained aneuploid cell populations (DI $<1$ or $>1$ ). The percentage of aneuploid cells is indicated in brackets.

\begin{tabular}{ccc}
\hline & TZ & PBZ \\
\hline GB-02 & $1+1.97(13.5 \%)$ & $\mathbf{1 ( 0 \% )}$ \\
\hline GB-03 & $1+0.95(56 \%)$ & $\mathbf{1}(\mathbf{0} \%)$ \\
\hline GB-04 & $1+0.9+1.8(18 \%)$ & $1+0.9(24 \%)$ \\
\hline GB-05 & $1+0.9+1.8(48 \%)$ & $1+0.9+1.8(30 \%)$ \\
\hline GB-06 & $1+1.8(29 \%)$ & $1+1.8(3 \%)$ \\
\hline GB-07 & $1+2+2.3(78 \%)$ & $\mathbf{1 ( 0 \% )}$ \\
\hline GB-08 & $1+1.43(76 \%)$ & $\mathbf{1 ( 0 \% )}$ \\
\hline GB-09 & $1+1.54+1.7(57 \%)$ & $1+1.7(3 \%)$ \\
\hline GB-10 & $1+0.9+1.8(57 \%)$ & $1+0.9(44 \%)$ \\
\hline GB-11 & $1+1.7+1.8(21 \%)$ & $\mathbf{1 ( 0 \% )}$ \\
\hline GB-12 & $1+0.9+1.8(39 \%)$ & $1+0.9(20 \%)$ \\
\hline GB-15 & $1+1.05+2.05(55 \%)$ & $1+1.05+2.05(26 \%)$ \\
\hline GB-21 & $1+1.45(18 \%)$ & $\mathbf{1 ( 0 \% )}$ \\
\hline GB-29 & $1+1.04+2.01(84 \%)$ & $\mathbf{1 ( 0 \% )}$ \\
\hline GB-32 & $1+1.06+2.08(76 \%)$ & $\mathbf{1 ( 0 \% )}$ \\
\hline GB-34 & $1+0.96(22 \%)$ & $1+0.96(33 \%)$ \\
\hline GB-37 & $1+0.95(50 \%)$ & $\mathbf{1 ( 0 \% )}$
\end{tabular}

\section{Genomic analysis}

Array-CGH analysis of TZ showed genomic alterations commonly described in GB, such as gain of chromosome 7 with EGFR amplification, deletion of chromosome 10, and loss of CDKN2A/2B on chromosome 9 (Aubry et al., submitted).

Six of the ten PBZ analyzed (GB-03, GB-09, GB-16, GB-17, GB-25, and GB-26) showed less than $1 \%$ of genome aberrations. The other four PBZ samples (GB-10, GB-15, GB-35, and GB-36) exhibited various large genomic alterations that were also present in tumor samples from these patients (Figure 2A). Individual arrayCGH profiles for each PBZ sample are available as supplementary data. The results of genomic analysis were correlated with those from histopathological analysis. All of the PBZ that were characterized as abnormal by genomic analysis exhibited tumor cell infiltration on microscopic examination. All the PBZ with minimal genomic alteration $(<1 \%)$ did not show tumor cell infiltration on histopathological analysis, except for the PBZ of GB-17, which exhibited a mild tumor cell infiltration.

Similar to findings from the flow cytometry analysis, abnormal PBZ samples exhibited only a few of the genetic alterations present in their respective TZ sample. For example, the CDKN2A/2B deletion was present 
in almost all TZ whereas only one PBZ harbored this anomaly (GB-36). The deletion of chromosome 10 and the gain of chromosome 7 with the EGFR (7p11) amplification were found in both altered PBZ and their respective TZ.

\section{Transcriptomic analysis}

The hierarchical clustering of transcriptomic data from nine PBZ and TZ samples, and four control brain samples distinguished two main clusters: one cluster grouping the PBZ with the control brain samples and the TZ from GB-09, and a second cluster comprising the remaining TZ (Figure 2B).

The PBZ and the control brain cluster could be further divided into two subgroups. The first subgroup comprised the control brain samples and the PBZ from GB-09, GB-16, GB-25, and GB-26. These PBZ did not show tumor cell infiltration on histopathological analysis and displayed minimal genomic alterations. Only the PBZ from GB-09 presented a tumor-related flow cytometry profile characterized by a rare aneuploid cell population (3\%). The second subgroup, which clustered closer to the TZ sample cluster than the first subgroup, comprised the TZ from GB-09 and the PBZ from GB-03, GB-15, GB-17, GB-35, and GB-36. These PBZ displayed tumor cell infiltration on histopathology and/or an altered genomic profile, with the exception of GB03 PBZ.

These results indicate that tumor infiltration in the PBZ alters the transcriptomic profile of this region but is not sufficient for PBZ and control brain samples to form separate clusters in transcriptomic analysis. The interpatient heterogeneity of PBZ samples as well as the low number of samples analyzed did not allow us to identify genes that were differentially expressed between PBZ and control brain samples. Such genes could be markers of recurrence mechanisms. 
A

\begin{tabular}{|c|c|c|c|c|c|c|c|c|c|c|c|}
\hline $\begin{array}{l}\text { Genomic } \\
\text { alteration }\end{array}$ & Gene & GB-03 & GB-09 & GB-10 & GB-15 & GB-16 & GB-17 & GB-25 & GB-26 & GB-35 & GB-36 \\
\hline Chr 7 amp & & - & - & $\begin{array}{c}\text { PBZ \& } \\
T Z\end{array}$ & $\begin{array}{c}\text { PBZ \& } \\
\text { TZ }\end{array}$ & - & - & - & - & $\begin{array}{c}\text { PBZ \& } \\
T Z\end{array}$ & $\begin{array}{c}\mathrm{PBZ} \& \\
\mathrm{TZ}\end{array}$ \\
\hline Chr 10 del & & - & - & $\begin{array}{c}\mathrm{PBZ} \& \\
\mathrm{TZ}\end{array}$ & $\begin{array}{c}\text { PBZ \& } \\
T Z\end{array}$ & - & - & - & - & $\begin{array}{c}\mathrm{PBZ} \& \\
\mathrm{TZ}\end{array}$ & $\begin{array}{c}\mathrm{PBZ} \& \\
\mathrm{TZ}\end{array}$ \\
\hline $4 q 12$ & PDGFRA & - & - & - & - & PBZ & - & $T Z$ & - & - & - \\
\hline $7 q 11$ & EGFR & - & - & - & - & - & $\begin{array}{c}\text { PBZ \& } \\
T Z\end{array}$ & - & - & $\begin{array}{c}\text { PBZ \& } \\
\text { TZ }\end{array}$ & $\begin{array}{c}\text { PBZ \& } \\
T Z\end{array}$ \\
\hline $5 p 15.3$ & TERT & - & - & - & - & - & - & - & - & $\begin{array}{c}\mathrm{PBZ} \& \\
\mathrm{TZ}\end{array}$ & - \\
\hline $9 q 11-9 q 21$ & CDKN2A/2B & $\mathrm{TZ}$ & $\mathrm{TZ}$ & - & - & $\mathrm{TZ}$ & $\mathrm{TZ}$ & $\mathrm{TZ}$ & $\mathrm{TZ}$ & $\mathrm{TZ}$ & $\begin{array}{c}\text { PBZ \& } \\
\text { TZ }\end{array}$ \\
\hline $12 q 15$ & MDM2 & - & - & - & $\begin{array}{c}\text { PBZ \& } \\
\text { TZ }\end{array}$ & - & - & - & - & - & - \\
\hline
\end{tabular}

B

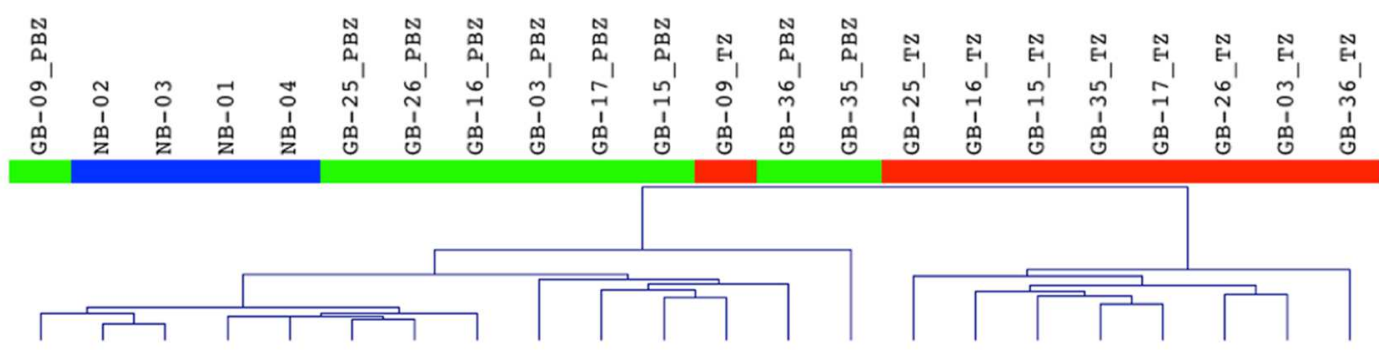

Fig. 2:

A) Most frequent genomic alterations found in the PBZ and the TZ.

B) Dendrogram of hierarchical clustering of brain samples based on the 1000 most differentially expressed genes between PBZ and TZ. Two main clusters of samples are observed: one cluster grouping the PBZ with the control brain samples (NB) and the TZ from GB-09, and a second cluster comprising the remaining TZ.

\section{Proteomic analysis}

We used the ICPL (isotope coded protein labeling) technique to examine the protein content of PBZ samples compared to their respective $\mathrm{TZ}$ samples and found that between 47 and $83 \%$ of proteins were differentially expressed between PBZ and TZ samples (Table 2). Similarly, a large proportion (44 to 83\%) of proteins were differentially expressed between PBZ samples and pooled samples from control brain (Table 2).

More detailed proteomic analyses are available in our previous studies [17, 18].

We assumed from these two comparative analyses that the PBZ can be considered as infiltrated if the number of differentially expressed proteins between the PBZ and the TZ is smaller than that between the PBZ and control brain. However, PBZ that were classified as infiltrated according to these parameters did not match those identified as infiltrated by histopathological examination. Thus, the PBZ harbored a proteomic profile distinct from that of $\mathrm{TZ}$ and control brain regardless of histological profile. As for the transcriptomic analysis, the identification of a proteomic profile specific to the PBZ was not possible and requires a larger cohort of samples. 
Table 2: Differential proteomic profile between PBZ and NB, and between PBZ and TZ

\begin{tabular}{cccccccc} 
& \multicolumn{3}{c}{ PBZ vs NB } & & \multicolumn{3}{c}{ PBZ vs TZ } \\
\cline { 2 - 4 } \cline { 7 - 9 } & IdProt & $\begin{array}{c}\text { Quant } \\
\text { Prot }\end{array}$ & Diff Prot & & IdProt & $\begin{array}{c}\text { Quant } \\
\text { Prot }\end{array}$ & Diff Prot \\
\hline GB-03 & 145 & 58 & $48(83 \%)$ & & 267 & 105 & $49(47 \%)$ \\
\hline GB-10 & 123 & 72 & $32(44 \%)$ & & 273 & 135 & $82(61 \%)$ \\
\hline GB-16 & 145 & 72 & $53(74 \%)$ & & 258 & 114 & $72(63 \%)$ \\
\hline GB-22 & 116 & 55 & $44(80 \%)$ & & 266 & 96 & $62(65 \%)$ \\
\hline GB-25 & 123 & 58 & $44(76 \%)$ & & 321 & 90 & $75(83 \%)$ \\
\hline GB-26 & 116 & 55 & $36(65 \%)$ & & - & - & - \\
\hline
\end{tabular}

NB: normal brain, IdProt: number of unique identified proteins, QuantProt: number of unique quantified proteins, DiffProt: number of unique quantified proteins that are differentially expressed between PBZ and the control sample. A threshold of $<0.71$ was used for under-expressed proteins and $>1.41$ was used for overexpressed proteins. Percentage of differentially expressed proteins (from the total number of unique quantified proteins) is displayed in brackets.

\section{Primary cultures}

We examined primary cultures of 19 samples of PBZ and TZ by phase-contrast microscopy. Six (32\%) of the PBZ cultures gave rise to tumor-like cells with a morphology similar to that of GB cells derived from their respective TZ sample (Figure 3, supplementary Table 2). These cells were spindle-shaped or clumped into irregular spheroids, often several layers thick. These PBZ samples, except for GB-11 PBZ, displayed tumor cell infiltration on histopathological and/or flow cytometry analyses. The other PBZ samples (68\%) devoid of tumor cells on histopathological and/or flow cytometry analyses gave rise to diploid cells that we called GB-associated stromal cells (GASCs). These cells were star-shaped, were unable to assemble into multiple-layered structures as shown for GB-03 (Figure 3), and could be maintained in culture until passage 10. We recently showed that these stromal cells, despite their normal genomic profile, may display tumorigenic properties similar to myofibroblasts or cancer-associated fibroblasts (CAFs) in carcinoma $[15,16]$. 


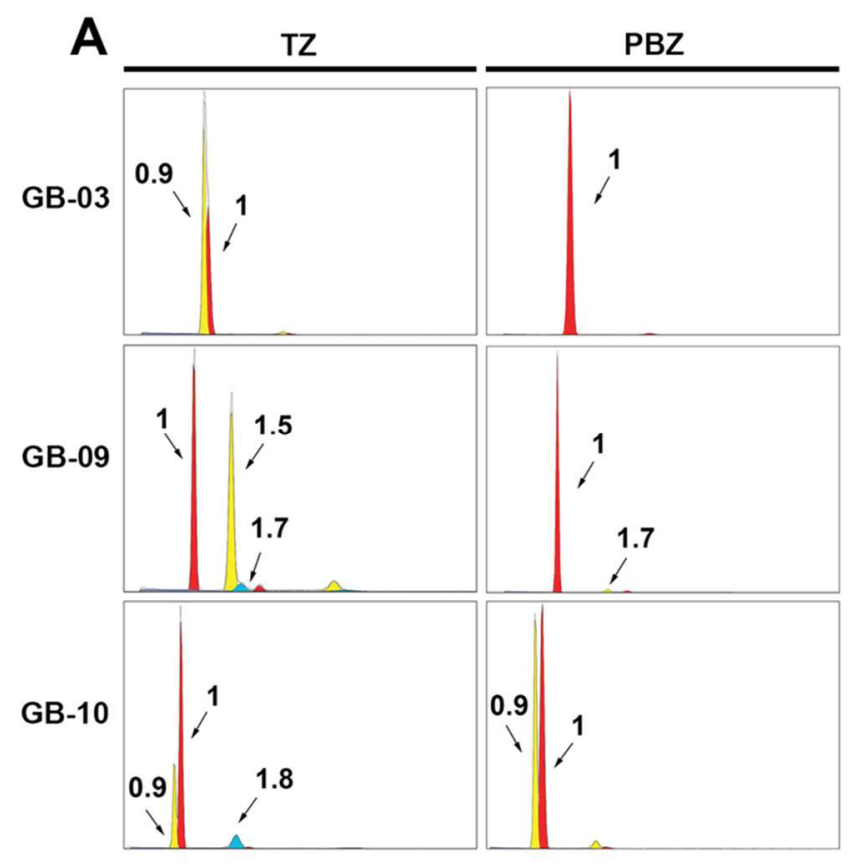

B
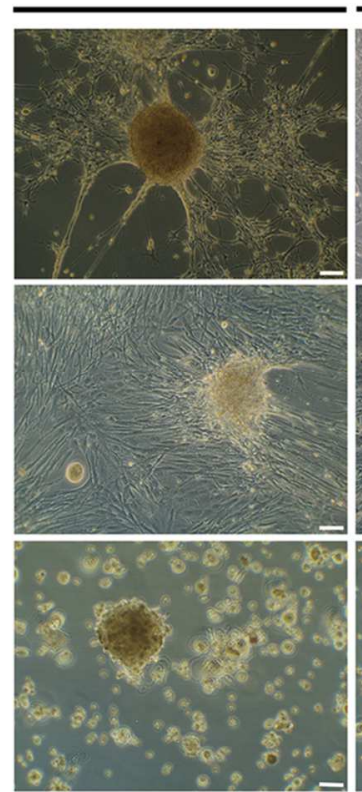

PBZ

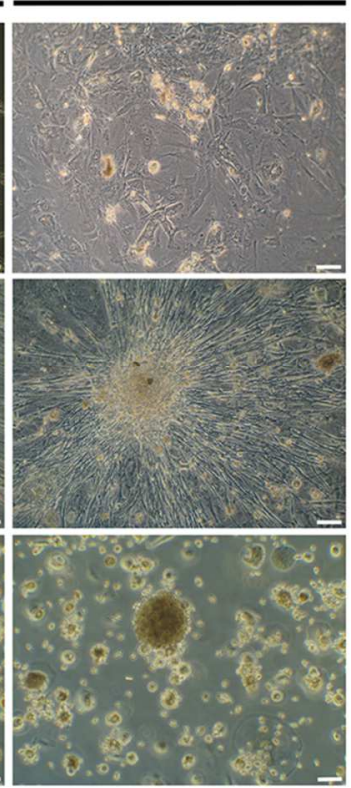

Fig. 3:

A) Flow cytometry histograms showing the DNA content of TZ biopsies (GB-03, GB-09 and GB-10) and their respective PBZ. A DI value of 1 was classified as DNA diploid and DI values of $<1$ or $>1$ were classified as aneuploid.

B) Phase-contrast microscopy of primary cultures of TZ biopsies (GB-03, GB-09 and GB-10) and their respective PBZ (P1 or P2). Cells derived from TZ cultures were spindle-shaped and clumped into irregular spheroids, often several layers thick. PBZ cultures from GB-09 and GB10, in which tumor cells were present to a significant level in the biopsy specimen before culture, these cells proliferated. In the PBZ culture from GB-03 in which tumor cells were absent, cell culture led, after two passages, to a diploid cell population. These diploid cells, which we called GASCs, were star-shaped and unable to assembly into multiple-layered structures. The bar indicates $50 \mu \mathrm{m}$.

\section{Discussion}

Although the PBZ is largely considered as abnormal, this study is the first to analyze the PBZ via a multidisciplinary approach, and paints a picture of a PBZ that is normal from afar but far from normal.

\section{Normal from afar ...}

Radiological and macroscopic analyses revealed that the PBZ resembled normal brain tissue. The PBZ presented a homogeneous DCE profile similar to that of normal brain but unlike that of the other sampling zones of GB. This radiological profile was characterized by low permeability and low extracellular volume fraction. This reflects the maintenance of the blood-brain barrier in the PBZ, which would explain the absence of contrast enhancement because the permeability in DCE imaging is proportional to the tumor histopathological grade [26]. However, radiological analysis of low-grade glioma has shown that tumor infiltration may be present without neoangiogenesis, thus without contrast enhancement and that the tumoral cell density threshold to modify the 
MRI signal must be around 500 cells $/ \mathrm{mm}^{3}$ [27], showing the limitations of radiological examination to apprehend glial tumor's infiltration in the brain parenchyma. This is highlighted by the results of histopathological examination that found tumor cell infiltration in one third of radiologically normal PBZ.

Other pre-surgical MRI techniques such as diffusion- and perfusion-weighted imaging and spectroscopy may be useful to evaluate tumor cell infiltration in PBZ. These approaches have already been used to assess the histological nature of the adjacent tumor [28-30] or the WHO grade of glial tumors [31, 32]. The authors of a recent study used diffusion tensor imaging sequences to assess PBZ infiltration and showed that vasogenic edema and tumor-infiltrated edema are characterized by distinct patterns in imaging data [33]. Further radiological studies that take into account this interesting result may be able to refine the pre-operative evaluation of PBZ infiltration, which may challenge traditional surgical strategies based on the removal of the contrast-enhanced tumor. In parallel, per-operative analysis of the resection margin, and thus of the PBZ, is currently increasingly performed during surgery, with the development of intra-operative CT or MRI and fluorescence-guided surgery with 5-aminolevulinic acid [5, 34, 35].

\section{... But far from normal}

As indicated above, histopathological examination found tumor cell infiltration in one third of PBZ in contrast with radiological and macroscopic analyses, which is consistent with data from the literature [9-11, 36]. Flow cytometry, genomic, transcriptomic, and primary cell culture approaches also identified these neoplastic features in these PBZ. Flow cytometry and primary culture analyses were more sensitive than histological analyses because they were able to identify tumor cell infiltration in histologically normal PBZ.

Proteomic and transcriptomic analyses showed that the RNA and protein content of the PBZ is distinct from that of both the TZ and control brain. However, due to the inter-patient heterogeneity of the PBZ, we were unable to identify specific markers that could be involved in recurrence mechanisms. A large cohort of PBZ samples is necessary to identify such markers, but the constitution of such a cohort faces the ethical issue of sampling "normal" brain tissue around the tumor. Furthermore, the identification of markers is also made difficult by the choice of the brain control sample to be used. Obviously, brain samples from healthy, living individuals are difficult to obtain and the control samples commonly used include tissue obtained during brain surgery for pathological conditions such as epilepsy, with the informed consent of the patient. However, we showed recently that epilepsy samples have a "tumoral" protein expression pattern [17]; thus, caution should be urged about the use of these samples as control samples. Post-mortem brain tissue obtained from autopsy is a potential, alternative source of brain control samples. However, RNA and several highly abundant proteins are 
degraded rapidly after death [37, 38]; therefore, the use of such control brain samples necessitates quick sampling and storage, which are difficult to implement. These observations show that it is a major obstacle to find brain control samples with a proteome and transcriptome that are similar to that normally present in vivo.

Although we have not identified representative markers of the PBZ through transcriptomic and proteomic analyses, we observed specific alterations of this area through genomic, flow cytometry and primary culture analyses. We show that the PBZ possesses some, but not all, of the tumor cell alterations found in the TZ. In particular, genomic analyses indicated that the deletion of chromosome 10 and the EGFR (7p11) amplification present in the $\mathrm{TZ}$ were also present in PBZ, whereas the CDKN2A/B deletion, which was also present in the TZ, was in most cases absent from the PBZ. The amplification of EGFR in the PBZ was also described by Mangiola et al. (2013) [39]. These results suggest that copy number alterations targeting chromosome 7 and 10 are among the earliest events in GB tumor evolution and that some tumor clones, but not all, migrate away from the tumor. Some studies have reported findings that support this suggestion [12-14]. For example, Glas et al. (2010) used primary cultures to isolate tumor cells from the brain tissue surrounding the resection cavity [12]. These infiltrating tumor cells displayed genetic alterations typical of GB, but could be distinguished from tumor cells isolated from the tumor core on the basis of their distinctive molecular profiles and responses to drugs and irradiation in vitro. Similarly, Piccirillo et al. (2012) used fluorescence-guided surgical sampling of GB to identify phenotypically distinct tumor-initiating cell populations in the tumor mass and margins [13]. Our observations in primary cultures show that the PBZ can be altered even in the absence of tumor cell infiltration. We isolated a population of stromal cells that we named GASCs, which share phenotypic and functional properties with CAFs described in the stroma of carcinomas. In particular, they have a myofibroblast phenotype and tumorigenic and angiogenic properties $[15,16]$. GASCs may be implicated in recurrence mechanisms of GB as infiltrating tumor cells.

\section{Conclusion}

This multidisciplinary analysis of the PBZ confirms that macroscopic and radiological analyses are insufficient to determine whether the PBZ has an abnormal profile. Omic analyses highlight the complexity of this zone, which shows inter-patient variability similar to its corresponding tumor zone. This complexity makes it difficult to identify representative markers of the PBZ. The observation of select tumor clones in the PBZ and the presence of stromal cells with tumorigenic and angiogenic properties emphasizes the importance to characterize better this area in order to: 1) develop approaches to refine the per-operative evaluation of the PBZ 
to optimize the surgical resection of the tumor, and 2) improve the understanding of mechanisms that underlie

GB recurrence to develop new therapies.

\section{Acknowledgements}

We gratefully acknowledge the neurosurgeons, the radiologists and the neuropathologists at the University Hospitals of Angers, Rennes, Poitiers, Brest, and Tours for supplying us with GB and PBZ tissue samples. We also thank the members of the glioma network of the Cancéropole Grand Ouest and Agnès Chassevent for providing facilities, and A. Edelman and Associates for correcting the manuscript.

\section{Disclosure}

The authors report no conflict of interest concerning the materials or methods used in this study or the findings specified in this paper.

\section{References}

1. Stupp R, Hegi ME, Mason WP, et al (2009) Effects of radiotherapy with concomitant and adjuvant temozolomide versus radiotherapy alone on survival in glioblastoma in a randomised phase III study: 5year analysis of the EORTC-NCIC trial. Lancet Oncol 10:459-466

2. Stupp R, Mason WP, van den Bent MJ, et al (2005) Radiotherapy plus concomitant and adjuvant temozolomide for glioblastoma. N Engl J Med 352:987-996

3. Chaichana KL, Jusue-Torres I, Navarro-Ramirez R, et al (2013) Establishing percent resection and residual volume thresholds affecting survival and recurrence for patients with newly diagnosed intracranial glioblastoma. Neuro-Oncol. doi: 10.1093/neuonc/not137

4. Orringer D, Lau D, Khatri S, Zamora-Berridi GJ, Zhang K, Wu C, Chaudhary N, Sagher O (2012) Extent of resection in patients with glioblastoma: limiting factors, perception of resectability, and effect on survival. J Neurosurg 117:851-859

5. Stummer W, Reulen H-J, Meinel T, et al (2008) Extent of resection and survival in glioblastoma multiforme: identification of and adjustment for bias. Neurosurgery 62:564-576; discussion 564-576

6. McGirt MJ, Chaichana KL, Gathinji M, Attenello FJ, Than K, Olivi A, Weingart JD, Brem H, QuiñonesHinojosa AR (2009) Independent association of extent of resection with survival in patients with malignant brain astrocytoma. J Neurosurg 110:156-162

7. Sherriff J, Tamangani J, Senthil L, Cruickshank G, Spooner D, Jones B, Brookes C, Sanghera P (2013) Patterns of relapse in glioblastoma multiforme following concomitant chemoradiotherapy with temozolomide. Br J Radiol 86:20120414

8. Petrecca K, Guiot M-C, Panet-Raymond V, Souhami L (2013) Failure pattern following complete resection plus radiotherapy and temozolomide is at the resection margin in patients with glioblastoma. $\mathrm{J}$ Neurooncol 111:19-23 
9. Nagashima G, Suzuki R, Hokaku H, Takahashi M, Miyo T, Asai J, Nakagawa N, Fujimoto T (1999) Graphic analysis of microscopic tumor cell infiltration, proliferative potential, and vascular endothelial growth factor expression in an autopsy brain with glioblastoma. Surg Neurol 51:292-299

10. SCHERER HJ (1940) THE FORMS OF GROWTH IN GLIOMAS AND THEIR PRACTICAL SIGNIFICANCE. Brain 63:1-35

11. Yamahara T, Numa Y, Oishi T, Kawaguchi T, Seno T, Asai A, Kawamoto K (2010) Morphological and flow cytometric analysis of cell infiltration in glioblastoma: a comparison of autopsy brain and neuroimaging. Brain Tumor Pathol 27:81-87

12. Glas M, Rath BH, Simon M, et al (2010) Residual tumor cells are unique cellular targets in glioblastoma. Ann Neurol 68:264-269

13. Piccirillo SGM, Dietz S, Madhu B, Griffiths J, Price SJ, Collins VP, Watts C (2012) Fluorescence-guided surgical sampling of glioblastoma identifies phenotypically distinct tumour-initiating cell populations in the tumour mass and margin. Br J Cancer 107:462-468

14. Ruiz-Ontañon P, Orgaz JL, Aldaz B, et al (2013) Cellular plasticity confers migratory and invasive advantages to a population of glioblastoma-initiating cells that infiltrate peritumoral tissue. Stem Cells Dayt Ohio 31:1075-1085

15. Clavreul A, Etcheverry A, Chassevent A, et al (2012) Isolation of a new cell population in the glioblastoma microenvironment. J Neurooncol 106:493-504

16. Clavreul A, Guette C, Faguer R, et al (2014) Glioblastoma-associated stromal cells (GASCs) from histologically normal surgical margins have a myofibroblast phenotype and angiogenic properties. $\mathrm{J}$ Pathol. doi: 10.1002/path.4332

17. Lemée J-M, Com E, Clavreul A, Avril T, Quillien V, de Tayrac M, Pineau C, Menei P (2013) Proteomic analysis of glioblastomas: what is the best brain control sample? J Proteomics 85:165-173

18. Com E, Clavreul A, Lagarrigue M, Michalak S, Menei P, Pineau C (2012) Quantitative proteomic IsotopeCoded Protein Label (ICPL) analysis reveals alteration of several functional processes in the glioblastoma. J Proteomics 75:3898-3913

19. De Tayrac M, Etcheverry A, Aubry M, Saïkali S, Hamlat A, Quillien V, Le Treut A, Galibert M-D, Mosser J (2009) Integrative genome-wide analysis reveals a robust genomic glioblastoma signature associated with copy number driving changes in gene expression. Genes Chromosomes Cancer 48:55-68

20. De Tayrac M, Saikali S, Aubry M, Bellaud P, Boniface R, Quillien V, Mosser J (2013) Prognostic significance of EDN/RB, HJURP, p60/CAF-1 and PDLI4, four new markers in high-grade gliomas. PloS One 8:e73332

21. De Tayrac M, Aubry M, Saïkali S, et al (2011) A 4-gene signature associated with clinical outcome in highgrade gliomas. Clin Cancer Res Off J Am Assoc Cancer Res 17:317-327

22. Etcheverry A, Aubry M, de Tayrac M, et al (2010) DNA methylation in glioblastoma: impact on gene expression and clinical outcome. BMC Genomics 11:701

23. Louis DN, Ohgaki H, Wiestler OD, Cavenee WK, Burger PC, Jouvet A, Scheithauer BW, Kleihues P (2007) The 2007 WHO Classification of Tumours of the Central Nervous System. Acta Neuropathol (Berl) 114:97-109

24. Vindel $\varnothing v$ LL, Christensen IJ, Jensen G, Nissen NI (1983) Limits of detection of nuclear DNA abnormalities by flow cytometric DNA analysis. Results obtained by a set of methods for sample-storage, staining and internal standardization. Cytometry 3:332-339 
25. Tofts PS, Kermode AG (1991) Measurement of the blood-brain barrier permeability and leakage space using dynamic MR imaging. 1. Fundamental concepts. Magn Reson Med Off J Soc Magn Reson Med Soc Magn Reson Med 17:357-367

26. Zhang N, Zhang L, Qiu B, Meng L, Wang X, Hou BL (2012) Correlation of volume transfer coefficient Ktrans with histopathologic grades of gliomas. J Magn Reson Imaging JMRI 36:355-363

27. Jbabdi S, Mandonnet E, Duffau H, Capelle L, Swanson KR, Pélégrini-Issac M, Guillevin R, Benali H (2005) Simulation of anisotropic growth of low-grade gliomas using diffusion tensor imaging. Magn Reson Med Off J Soc Magn Reson Med Soc Magn Reson Med 54:616-624

28. Server A, Kulle B, Maehlen J, Josefsen R, Schellhorn T, Kumar T, Langberg CW, Nakstad PH (2009) Quantitative apparent diffusion coefficients in the characterization of brain tumors and associated peritumoral edema. Acta Radiol Stockh Swed 1987 50:682-689

29. Tsolaki E, Svolos P, Kousi E, Kapsalaki E, Fountas K, Theodorou K, Tsougos I (2013) Automated differentiation of glioblastomas from intracranial metastases using 3T MR spectroscopic and perfusion data. Int J Comput Assist Radiol Surg 8:751-761

30. Tsougos I, Svolos P, Kousi E, Fountas K, Theodorou K, Fezoulidis I, Kapsalaki E (2012) Differentiation of glioblastoma multiforme from metastatic brain tumor using proton magnetic resonance spectroscopy, diffusion and perfusion metrics at 3 T. Cancer Imaging Off Publ Int Cancer Imaging Soc 12:423-436

31. Server A, Kulle B, Gadmar ØB, Josefsen R, Kumar T, Nakstad PH (2011) Measurements of diagnostic examination performance using quantitative apparent diffusion coefficient and proton MR spectroscopic imaging in the preoperative evaluation of tumor grade in cerebral gliomas. Eur J Radiol 80:462-470

32. Law M, Yang S, Wang H, Babb JS, Johnson G, Cha S, Knopp EA, Zagzag D (2003) Glioma grading: sensitivity, specificity, and predictive values of perfusion MR imaging and proton MR spectroscopic imaging compared with conventional MR imaging. AJNR Am J Neuroradiol 24:1989-1998

33. Min Z, Niu C, Rana N, Ji H, Zhang M (2013) Differentiation of pure vasogenic edema and tumor-infiltrated edema in patients with peritumoral edema by analyzing the relationship of axial and radial diffusivities on 3.0T MRI. Clin Neurol Neurosurg 115:1366-1370

34. Colditz MJ, Jeffree RL (2012) Aminolevulinic acid (ALA)-protoporphyrin IX fluorescence guided tumour resection. Part 1: Clinical, radiological and pathological studies. J Clin Neurosci Off J Neurosurg Soc Australas 19:1471-1474

35. Idoate MA, Díez Valle R, Echeveste J, Tejada S (2011) Pathological characterization of the glioblastoma border as shown during surgery using 5-aminolevulinic acid-induced fluorescence. Neuropathol Off J Jpn Soc Neuropathol 31:575-582

36. Burger PC, Heinz ER, Shibata T, Kleihues P (1988) Topographic anatomy and CT correlations in the untreated glioblastoma multiforme. J Neurosurg 68:698-704

37. Koppelkamm A, Vennemann B, Lutz-Bonengel S, Fracasso T, Vennemann M (2011) RNA integrity in post-mortem samples: influencing parameters and implications on RT-qPCR assays. Int J Legal Med 125:573-580

38. Sköld K, Svensson M, Norrman M, Sjögren B, Svenningsson P, Andrén PE (2007) The significance of biochemical and molecular sample integrity in brain proteomics and peptidomics: stathmin 2-20 and peptides as sample quality indicators. Proteomics 7:4445-4456

39. Mangiola A, Saulnier N, De Bonis P, et al (2013) Gene expression profile of glioblastoma peritumoral tissue: an ex vivo study. PloS One 8:e57145 


\section{Supplementary methods}

\section{Radiological analysis}

Standardized sequences were used for pre-surgical MRI at each participating center and were carried out in the following order: T1-weighted axial and coronal acquisitions, diffusion, a quantitative dynamic contrastenhanced (DCE) session after the injection of gadoteric acid at a concentration of $0.1 \mathrm{mmol} / \mathrm{kg}$, and finally $\mathrm{T} 2$ FLAIR, T2-weighted and 3D T1 acquisitions.

Preliminary quality controls were performed at each center to ensure that acquisitions between the different sites were similar. Signal homogeneity, geometric distortions, spatial resolution, slice position, and MRI acquisition parameters were controlled between the different MR scanners ${ }^{29}$. Images were written onto a CDROM and transferred to the reference center for centralized interpretation (Functional imaging platform PRISM, Cancéropôle Grand Ouest, Rennes, France).

Quantitative and semi-quantitative DCE-MRI variables were calculated in all four zones (NZ, TZ, IZ and PBZ) for seven patients, with a DCE-MRI protocol adapted from a previous study ${ }^{11}$. 2D Spoiled Gradient Echo sequences $\left(\mathrm{TR} / \mathrm{TE}=150 / 5.6 \mathrm{~ms}\right.$ and variable flip angle, $\left.\theta 1=10^{\circ}, \theta 2=90^{\circ}\right)$ were performed. Thirteen sagittal slices were acquired with a field of view of $180 \mathrm{~mm}$ x $240 \mathrm{~mm}$, a slice thickness of $5 \mathrm{~mm}$, and a 192 x 256 matrix leading to spatial resolution of $0.94 \mathrm{~mm}$ x $0.94 \mathrm{~mm}$. Five longitudinal relaxation time (T1) calibration vials were positioned in the coil and were simultaneously imaged with the patient.

Images were corrected for head movements prior to DCE-MRI post-processing with 3D Slicer (www.slicer.org) and variables were calculated with Matlab (MATLAB and Statistics Toolbox Release 2012b, The MathWorks, Inc., Natick, MA, USA). We were able to extract several variables from the DCE-MRI curves; the transfer constant $\left(\mathrm{k}^{\text {trans }}\right)$, which reflects capillary permeability, the fractional volume $\left(\mathrm{v}_{\mathrm{e}}\right)$ of extravascular extracellular space, which indicates the tissue fraction accessible to the contrast agent, and other variables [s0, s30, s60, s360, the maximal relaxation rate variation $(\Delta \mathrm{R} 1 \mathrm{max})$, time to peak (TTP), the area under the curve (AUC), and the longitudinal relaxation time $\left.\left(\mathrm{T} 1_{0}\right)\right]$. The experimental data were adjusted with the Tofts experimental model to extract characteristics of the different sampling zones ${ }^{39}$.

Statistical analysis was carried out with ANOVA for multivariate analysis and post-hoc analysis was carried out with an LSD test and a T3 Dunnett's test. Alpha risk was set at $5 \%$.

\section{Genomic and transcriptomic analyses}


PBZ and TZ samples from ten patients were used for genomic analyses and samples from nine patients were used for transcriptomic analyses. We also used four samples of control brain parenchyma that were obtained from patients who underwent cortectomy for epilepsy, as a control for transcriptomic analysis. Total DNA was isolated with Nucleospin tissue kit and total RNA was isolated with the NucleoSpin RNAII kit (both from Macherey-Nagel, Hoerdt, France). DNA quality was assessed by electrophoresis in a $1 \%$ agarose gel. RNA integrity (RNA integrity NC8) was confirmed with an Agilent 2100 bioanalyzer (Agilent Technologies, Santa Clara, CA, USA).

The full procedure for the analysis of genomic data is described in one of our previous publications ${ }^{9}$. Briefly, genomic alteration profiles were obtained by comparing copy number variation between DNA extracted from the sampling zones and matching constitutional DNA by HaarSeg and CGHcall methods with waviCGH software ${ }^{2}$. Comparisons between PBZ and TZ copy number alteration profiles were extracted from the TuMult analysis performed with all the samples included in the original study ${ }^{19}$. Analysis of gene expression profiles was restricted to $\mathrm{TZ}$ and PBZ and was performed with $\mathrm{TMeV}$ software ${ }^{30}$. Probes were selected on the basis of their expression levels (intensity greater than 100 in at least one of the two conditions). The 1000 most differentially expressed genes between PBZ and TZ were identified by a two-class paired SAM (Significant Analysis of Microarray). PBZ, TZ, and CB samples were grouped by hierarchical clustering with average linkage and Euclidean distance.

\section{Proteomic analysis}

The isotope-coded protein labeling technique (ICPL) was used to analyze PBZ and TZ samples from six patients and the proteomic profile of PBZ was compared to that of TZ. Pooled brain samples from three patients who underwent epilepsy surgery were used as control brain samples. During the ICPL method, intact proteins are labeled with isotopic derivatives of nicotinic acid of different molecular weights; this is followed first by gel liquid chromatography and subsequently by tandem mass spectrometry (GeLC-MS/MS) to identify and quantify proteins with an Esquire HCT Ultra PTM Discovery mass spectrometer. Detailed methodological information about ICPL is available in our previous publications ${ }^{7,18}$.

Peptides were identified by querying the human Swiss-Prot database with the Mascot search engine (v2.2.07), applying a score above the identity threshold and a false discovery rate (FDR) $<1 \%$. The ProteinExtractor algorithm in the ProteinScape 2.0 software was used to compile the identified peptides into a non-redundant protein list. Relative quantification of labeled peptides was determined for each sample with the 
WarpLC 1.2 software. The threshold of differential expression between PBZ and CB samples, and between PBZ and $\mathrm{TZ}$ samples, was set to $>1.41$ for up-regulated proteins and $<0.71$ for down-regulated proteins, which is above the calculated technical variation of the method ${ }^{7}$.

\section{Références}

1. Eliat P-A, Olivié D, Saïkali S, Carsin B, Saint-Jalmes H, de Certaines JD. Can dynamic contrast-enhanced magnetic resonance imaging combined with texture analysis differentiate malignant glioneuronal tumors from other glioblastoma? Neurol Res Int. 2012;2012:195176.

2. Tofts PS, Kermode AG. Measurement of the blood-brain barrier permeability and leakage space using dynamic MR imaging. 1. Fundamental concepts. Magn Reson Med Off J Soc Magn Reson Med Soc Magn Reson Med. 1991 Feb;17(2):357-67.

3. De Tayrac M, Etcheverry A, Aubry M, Saïkali S, Hamlat A, Quillien V, et al. Integrative genome-wide analysis reveals a robust genomic glioblastoma signature associated with copy number driving changes in gene expression. Genes Chromosomes Cancer. 2009 Jan;48(1):55-68.

4. Carro A, Rico D, Rueda OM, Díaz-Uriarte R, Pisano DG. waviCGH: a web application for the analysis and visualization of genomic copy number alterations. Nucleic Acids Res. 2010 Jul;38(Web Server issue):W182-187.

5. Letouzé E, Allory Y, Bollet MA, Radvanyi F, Guyon F. Analysis of the copy number profiles of several tumor samples from the same patient reveals the successive steps in tumorigenesis. Genome Biol. 2010;11(7):R76.

6. Saeed AI, Sharov V, White J, Li J, Liang W, Bhagabati N, et al. TM4: a free, open-source system for microarray data management and analysis. BioTechniques. 2003 Feb;34(2):374-8.

7. Com E, Clavreul A, Lagarrigue M, Michalak S, Menei P, Pineau C. Quantitative proteomic Isotope-Coded Protein Label (ICPL) analysis reveals alteration of several functional processes in the glioblastoma. J Proteomics. 2012 Jul 16;75(13):3898-913.

8. Lemée J-M, Com E, Clavreul A, Avril T, Quillien V, de Tayrac M, et al. Proteomic analysis of glioblastomas: what is the best brain control sample? J Proteomics. 2013 Jun 24;85:165-73. 